\title{
MÁS ALLÁ DE PIAGET: COGNICIÓN ADULTA Y EDUCACIÓN
}

\section{Beyond Piaget: adult cognition and education}

\author{
Antonio Víctor MARTÍN GARCÍA \\ Universidad de Salamanca. Facultad de Educación. Departamento de Teoria e \\ Historia de la Educación. Paseo de Canalejas, 169. 37003 Salamanca.
}

Fecha de aceptación definitiva: marzo de 2000.

BIBLID [(1130-3743) 11, 1999, 127-157]

RESUMEN

El objetivo de este artículo es intentar una aproximación a la descripción del tipo de pensamiento que utilizan los adultos. Partimos de la base en nuestro trabajo de que el conocimiento sobre el modo como piensan las personas adultas se presenta como un aspecto clave para la teoría pedagógica y especialmente para la práctica, cuando se orienta hacia este tipo de alumnos. Para ello, organizamos el texto integrando el concepto de pensamiento en un constructo más amplio y comprensivo como es el de cognición. Desde ese marco realizamos, en primer lugar, una síntesis crítica de la teoría piagetiana en lo referente al desarrollo cognitivo por cuanto representa el modelo, ya clásico, más elaborado sobre el tema, y en segundo, un análisis de algunas de las formulaciones teóricas y empíricas recientes más importantes sobre la cognición adulta, como por ejemplo las derivadas del procesamiento de la información, de la perspectiva del Life-span, o del denominado modelo de encapsulación. Terminamos intentando ofrecer el estado de la cuestión en relación con los aspectos nucleares del pensamiento adulto. Todo ello con vistas a apoyar procesos de intervención e investigación educativa en este campo.

SUMMARY

The aim this article is to attempt an approach to a description of the type of thought that adults use. We take for granted in our paper that knowledge about the 
way adults think is a key aspect in pedagogical theory and especially in terms of practice when it is directed towards this type of students. With this in mind we organize the text integrating the concept of thought in a wider an more comprehensive construct which is cognition. From this framework we make, first of all, a critical synthesis of the Piaget theory related to cognitive development in as far as it represents the now classical model, but more developed on the subject and, secondly an analysis of some of the most important of the recent theoretical and empirical formulation of adult cognition, such as those derived from information process and from the Lifespan perspective. We finish attempting to offer the state of the question in relation to nuclear aspects of adult thought. The aim of all this is to support processes of educational intervention and research in this field.

\section{INTRODUCCIÓN: LA COGNICIÓN ADULTA}

Recientemente, con motivo del aniversario del centenario del nacimiento de Jean Piaget se acentuaron los análisis sobre su obra, desplegándose monográficos y números especiales en revistas científicas en las que se discutió sobre la vigencia o no de su teoría, artículos que se sumaron a las múltiples revisiones y homenajes de reconocimiento en todo el mundo que ya se efectuaron en el año de su muerte en 1980. El amplio acuerdo sobre el impacto de los trabajos de Jean Piaget y la Escuela de Ginebra es aplicable no sólo al desarrollo cognitivo infantil y adolescente, sino que muchos especialistas consideran que, especialmente la formulación de Inhelder y Piaget (1958) sobre las operaciones formales, ha influido profundamente en el estudio del desarrollo cognitivo adulto de los últimos 25 años. En general, como apunta Patricia King (1986) los conceptos teóricos, metodologías y aplicaciones educativas de Piaget han servido para estimular la imaginación colectiva de miles de teóricos, investigadores, educadores y profesores de todo el mundo. Lo interesante es que estos estudios prosiguen hoy bajo diferentes formas dentro de la corriente organísmica, tratando de mantener muchas de las ideas piagetianas, o readaptando otras, buscando en suma nuevas explicaciones sobre la cognición adulta.

Nuestra pretensión en este artículo no es ampliar o revisar lo revisado sobre la perspectiva organísmica ${ }^{1}$, sino, en todo caso, reconsiderar una de las líneas de

1. Puede verse al respecto: las revistas Substratum (1996). Cien años con Piaget, III (8-9); Anuario de Psicología no 6 (1996); Infancia y Aprendizaje (1981). Piaget. Monografía. Por poner algún ejemplo, H. Gardner (1993:43), bajo el epígrafe "la mente después de Piaget" señala la importancia de la obra de Piaget, no sólo entre los neopiagetianos, incluso entre aquellos que encuentran faltas entre sus formulaciones. En la misma línea y en el campo de la psicología española, César Coll hacía un análisis recientemente sobre el impacto de la teoría genética en el pensamiento epistemológico y educativo contemporáneo viniendo a reiterar la idea de que "resulta difícil dejar de tener en cuenta las ideas de Jean Piaget incluso a los que no las comparten "..." hoy por hoy, la teoría genética sigue ejerciendo un atractivo innegable e interpelando con fuerza a epistemólogos, psicológos, especialistas en educación y otros científicos (Coll, 1996: 213). 
trabajo teórico y de investigación que se inició hace algo más de una década y que pretendía ir, como señalaba Broughton en 1984, "más allá de Piaget”. Esto es, continuar los trabajos iniciados por Piaget y colaboradores manteniendo los aspectos centrales de su teoría. Efectivamente, aunque la diversidad de revisiones críticas, de modelos y teorías alternativas a los postulados piagetianos sobre las operaciones formales ha generando bastante confusión, fundamentalmente por su falta de concreción conceptual y ausencia de consistencia empírica, sin embargo, a principios de los años 80 comenzaron a apuntalar una prometedora línea teórica y de investigación que, en nuestra opinión mantiene un notable interés para la teoría y práctica educativa con personas adultas y mayores.

La emergencia de la adultez y de la vejez como centro de interés social y académico ha contribuido a dar un notable impulso al desarrollo de la investigación (fundamentalmente psicológica) sobre el desarrollo de las funciones cognitivas en estas etapas del ciclo humano. En el caso particular de la cognición, el interés responde básicamente a la necesidad de conocer tanto el potencial de desarrollo cognitivo de los individuos, como los efectos del deterioro o la pérdida de funcionalidad del mismo y de sus funciones asociadas. La búsqueda de conocimiento que ayude a comprender qué implicaciones puede tener el buen o mal funcionamiento cognitivo sobre nuestros sistemas de atribuciones y autopercepciones (por ejemplo, en las creencias sobre el mundo, sobre nosotros mismos o en las valoraciones a que conducen en contacto con el medio y la interacción social), sobre el conocimiento de nuestros propios procesos cognitivos (metacognición) o, en definitiva, sobre las posibilidades de aprendizaje a cualquier edad, es de un extraordinario interés en sí mismas y lo es para la teoría y práctica educativa, y se ha erigido en un vector que está guiando a los diferentes grupos de investigadores sobre el tema. Gran parte de esa investigación ha tomado como punto de partida la revisión de muchos de los principios piagetianos sobre el desarrollo cognitivo, buscando identificar aquellos aspectos que cognitivamente permitan distinguir a un adulto de un adolescente.

El desarrollo de toda esa serie de planteamientos, con un marcado carácter revisionista y en muchos casos también críticos sobre la teoría genética, se reúnen dentro de lo que se ha dado en llamar "el pensamiento postformal", o también, "los estilos postformales de pensamiento", bien entendido que estos estilos de pensamiento se encuentran incardinados dentro de un proceso más global e integrador que aparece rotulado con el término cognición.

De entrada resulta necesario destacar el carácter multifacético y complejo de lo que se da en llamar cognición. La mayor parte de especialistas entienden que el significado de la cognición alude al conjunto de actividades a través de las cuales la información es procesada por el sistema psíquico. Se acepta así que el término cognición comprende toda una serie de procesos mentales que realizan los seres humanos para adquirir, retener, interpretar, comprender, organizar, utilizar y reutilizar tanto la información existente en el medio que nos rodea, como la propia información ya adquirida y almacenada. De este modo, la cognición incluye los 
procesos de percepción, atención, cognición espacial, imaginación, lenguaje, memoria, resolución de problemas, creatividad, pensamiento e inteligencia. Pero no sólo los procesos cognitivos sirven para procesar información, también para construir representaciones de la realidad y crear conocimiento. Es decir, el término se refiere tanto al sistema de procesamiento de la información como al contenido procesado y al resultado del proceso, es decir, al conocimiento. Esta visión integrada del concepto es fácilmente observable en algunas líneas recientes sobre el desarrollo cognitivo adulto como por ejemplo la teoría de la encapsulación de Rybas, Hoyer y Roodin formulada inicialmente en 1986, la teoría tridimensional de Stenberg (1992) o, en menor medida, la teoría sobre el desarrollo intelectual adulto de Ackerman (1996), en las que se conceptualiza la cognición como un proceso que integra tres aspectos dominantes como son el procesamiento, el conocimiento y el pensamiento.

Con todo, la dificultad a la hora de conceptualizar este constructo ha hecho que en muchas ocasiones el término haya sido utilizado de manera indistinta con los de inteligencia o pensamiento. La perspectiva psicométrica, que centró su interés más en los productos que en los procesos del desarrollo intelectivo adulto, representa el ejemplo clásico más claro de identificación de los conceptos inteligencia y cognición. El reduccionismo se ha dado aún en mayor medida en la identificación de los conceptos 'pensamiento' y 'cognición'. Como señala Rebok (1987) al respecto, aunque tradicionalmente las actividades diarias tales como atender, recordar y resolver problemas se describen bajo el concepto de pensamiento, éste es un concepto vago e impreciso, y la comprensión de estas actividades y procesos se expresan mejor mediante el término cognición.

Retomando el hilo conductor de este artículo, puede decirse que actualmente se distinguen dos líneas de trabajo a partir de la psicología genética piagetiana: por un lado, lo que podríamos denominar como nuevas elaboraciones sobre el pensamiento formal y, por otro, el desarrollo de modelos recientes sobre el denominado pensamiento post-formal. Naturalmente entre una y otra se han desarrollado otras alternativas y modelos propios sobre el desarrollo cognitivo adulto, algunas de las cuales con entidad sobradamente reconocida como son las del procesamiento de la información o la corriente de la psicología del desarrollo del ciclo vital, a las que nos referiremos también más adelante por su importancia para la definición y caracterización de la cognición adulta.

1.1. La Perspectiva organísmica sobre el desarrollo cognitivo adulto: una breve síntesis

Como se sabe, la teoría organísmica, respresentada por autores como Werner ${ }^{2}$, y muy especialmente Piaget y colaboradores, responde a una concepción interac-

2. La teoría de Heinz WERNER está estrechamente vinculada a la biología. También propone una serie de etapas del desarrollo o niveles (desarrollo sensoriomotor- desarrollo perceptual y desarrollo 
cionista según la cual el organismo construye sus propios esquemas de conocimiento en relación con el medio que le rodea. Como señala Rebok (1987) el enfoque organísmico asume que el proceso de cambio cognitivo es similar al proceso orgánico: de igual modo que el proceso biológico (respirar, comer, dormir...) nos capacita para adaptarnos y sobrevivir en un mundo en permanente cambio, los procesos cognitivos (memoria, resolución de problemas, inteligencia...) están organizados o estructurados para ejecutar una función adaptativa similar. Esta disposición adaptativa responde a la forma de conjunto o todo organizado (más que a un conjunto de partes constituyentes) que se encuentra siempre en estado de transición, desde un nivel de desarrollo cualitativamente inferior a otro superior, en una secuencia establecida de estadios.

La idea de estadio en la teoría genética implica que el desarrollo cognitivo esta dividido en períodos cualitativamente diferentes. Cada estadio representa un nivel distintivo de organización psicológica. La noción de estadio se encuentra asociada a la idea de discontinuidad en el desarrollo (es decir la aparición de características distintivas, diferentes a las de otros estadios anteriores o posteriores), a la idea de invariabilidad (los estadios son invariantes, es decir, proceden en un orden regular, no es posible regresar a una etapa anterior), así como a la idea de universalidad en el curso evolutivo (ocurren en la misma secuencia, en todas las cultura y para todos los individuos. En este sentido, a pesar que los individuos de diferentes culturas pueden diferir en el contenido de su pensamiento y puedan no alcanzar el final del estadio de desarrollo, sus habilidades cognitivas cambian en el mismo modo general) ${ }^{3}$.

La idea de emergencia de estructuras de conocimiento, que una vez que aparecen están en la mente y se estabilizan hasta la consolidación de una nueva estructura que subsume y reorganiza la anterior, va asociada a la noción de que todo avance significativo en el desarrollo cognitivo es el resultado de alguna forma de equilibración. El equilibrio de los sistemas cognitivos para Piaget se aproxima a la idea de un estadio estacionario pero dinámico (homeorresis) que es a la vez abierto y cerrado y que se origina como consecuencia de dos tipos de procesos constructivos: la asimilación y la acomodación (Piaget, 1990/1975) ${ }^{4}$. De este modo, para Piaget el aprendizaje, en términos generales, consistiría en el progreso de las estructuras cognitivas mediante procesos de equilibración, de modo que el apren-

contemplativo), aunque no tan bien especificadas ni conocidas como las de Piaget. Puede verse en: WERNER, H. (1957). The concept of development from a comparative and organismic point of view. En D.B. HARRIS (De.): The concept of development. Minneapolis: University of Minnesota Press.

3. Para una revisión sobre el concepto de estadio en Piaget puede verse PÉREZ PEREIRA, M. y DOSIL MACEIRA, A. (1994). El problema de los estadios en el desarrollo, en BERMEJO, V. (Ed.): Desarrollo cognitivo. Madrid: Síntesis. pp. 49-62.

4. PIAGET, J. (1990). La equilibración de las estructuras cognitivas. Problema central del desarrollo. Madrid: siglo XXI. (2. ed.) v.o. Piaget, J. (1975). L'equilibration des estructures cognitives. París: Presses Universitaires de France. 
dizaje se produce cuando se da un desequilibrio o un conflicto cognitivo entre estos dos procesos de construcción estática del conocimiento (en el sentido de que la nueva información se asimila a las estructuras de conocimiento ya existentes) y la construcción dinámica o, en términos piagetianos, acomodación, que es verdaderamente la que permite mediante determinados procesos modificar y "construir" el conocimiento (Pozo, 1996).

En todo este proceso, el papel de los organismos es fundamental dado que son activos en sus construcciones sobre el conocimiento a lo largo de las interacciones que mantienen con el ambiente, generando así nuevas o emergentes formas de desarrollo. Desde esta perspectiva constructivista se entiende que el organismo está estructurado físicamente y psicológicamente para alcanzar un punto final, un estadio final de desarrollo. En términos de crecimiento físico individual, el estado final se alcanza en la madurez biológica en la última fase de la adolescencia. De manera similar, el estadio final del desarrollo cognitivo, aunque más impreciso y complejo, se alcanzaría con el pensamiento abstracto, ejemplificado en el desarrollo de las operaciones formales 5 .

Hecha esta pequeña síntesis conceptual sobre la perspectiva organísmica, nos interesa analizar algunos de los resultados recientes que resumen el estado actual de esta línea de investigación, y que se podrían sintetizar en los siguientes puntos:

- A pesar del inicial carácter universal de todos los estadios, las operaciones formales son el punto más alto del desarrollo intelectual, pero no es alcanzado por todos los adultos, al menos no en todas las áreas.

- Las operaciones formales pueden describir sólo un aspecto del desarrollo cognitivo (por ejemplo, la habilidad para utilizar el razonamiento hipotético-deductivo), pero no explica plenamente el razonamiento en otros dominios (por ejemplo, en el terreno de la ética o la epistemología) que pueden ser desarrollados secuencialmente, pero de modo asincrónico con las operaciones formales (Mines y Kitchener, 1986: XI-XII).

- Frente a la idea extendida durante mucho tiempo de que los adultos mayores pierden las capacidades operatorias concretas y formales en sentido inverso a su adquisición (es decir, primero se perderían las operaciones formales, luego la conservación del volumen, peso, etc.) conocida como «bipótesis de la regresión", se admite hoy día su falta de consistencia empírica, debido a serios problemas metodológicos. Por ejemplo, el hecho de considerar como pérdida algo que no se ha llegado a adquirir, o el sesgo metodológico que introduce el recurso y abuso de la utilización de ancianos institucionalizados, a lo que habría que añadir problemas de salud y los efectos

5. Los múltiples estudios replicando los descubrimientos de Piaget, llevaron a éste a una revisión de la teoría, retrasando la plena aparición de las operaciones formales al periodo comprendido entre los 15 y 20 años, condicionando su aparición a la riqueza de la estimulación cognitiva que el entorno hubiese facilitado al niño (Vid. A. Clemente, 1996). 
generacionales y educativos, los factores motivacionales, los atributos de la pruebas, la homogeneidad y comparabilidad de las muestras..., etc.

- A diferencia del pensamiento operacional, el pensamiento formal admite que lo real es un subconjunto de lo posible, utiliza el razonamiento bipotético-deductivo y el pensamiento proposicional y permite al individuo no sólo clasificar o seriar objetos o eventos, sino también construir proposiciones acerca de esos datos y explorar caminos o vías en que están lógicamente relacionadas; en definitiva, amplía el marco de solución de determinados problemas (King, 1986) ${ }^{6}$. Sin embargo, no explica una nueva operación mental que es el descubrimiento de problemas, que se considera podría corresponder a un estado más avanzado (Arlin, 1986) ${ }^{7}$.

- Por otro lado, y a pesar de que la teoría piagetiana mantiene que existirían estructuras cognitivas comunes a todos los individuos, éstas podrían aplicarse diferencialmente a numerosas actividades del individuo, en particular aquéllas que se encuentran ligadas a nuevas tareas o a capacidades profesionales. Desde este punto de vista es desde el que se habla de babilidades ligadas a tareas especificas y ha llevado a estudiar ese proceso de diferenciación que hace que unos individuos adultos puedan desarrollar con una alta competencia, unas cápacidades (por ejemplo habilidades profesionales y de vida diaria) y no otras.

- En suma, se considera que las operaciones formales son un paso en la secuencia del desarrollo intelectual, pero no necesariamente el último. Y por otro lado, algunos de estos resultados coinciden en destacar las influencias de variables como el tiempo histórico y ambiental que explicarían las secuencias diferenciales del desarrollo.

A pesar de los avances y nuevos seguidores que se han sumado a esta línea de trabajo, lo cierto es que la perspectiva organísmica sigue presentando numerosas insuficiencias, algunas de las cuales resumimos a continuación.

6. Vid. Patricia M. KING "Formal reasoning in adults: a review and critique". En Mines y Kitchener (1986). Esta misma idea la recogen también Rybash, Hoyer y Roodin (1986) cuando señalan que, en general, existen tres características especiales asociadas con el pensamiento operacional formal (Inhelder y Piaget, 1958; Piaget y Inhelder, 1969). 1은 Los pensadores formales son capaces de desarrollar el pensamiento hipotético-deductivo. En otras palabras, los pensadores formales razonan como científicos, de manera que son capaces de comprobar hipótesis abastractas para observar la ocurrencia o no ocurrencia de resultados específicos. Esto es, construyen teorías que pueden ser comprobadas por la experimentación. $2^{\circ}$ Los pensadores formales son capaces de revertir las relaciones entre la realidad y la posibilidad. $3^{\circ}$ El pensador formal es capaz de pensar acerca del pensamiento.

7. Patricia Kennedy ARLIN: "Problem finding and young Adult Cognition". En Mines y Kitchener (1986), op. cit. pp. 22-32. 
1.2. Críticas a las operaciones formales formuladas por Piaget

$1^{\circ}$. Una crítica clásica es la que mantiene que el modelo carece de parsimonia (cf. Brainerd, 1978) y ajuste empírico (cf. Bynum, Thomas y Weitz, 1972). En el fondo subyace la idea en estas críticas de que la competencia lógica no puede ser detectada en la ejecución de los sujetos adolescentes. Consecuentemente, un modelo menos elaborado es suficiente para explicar el pensamiento adolescente (vid. Commoms, Richards y Armon, 1984).

$2^{\circ}$. Se le ha criticado también por fijar el proceso de desarrollo del pensamiento humano en los albores de la adultez temprana, concediendo escasa atención a los cambios durante el período adulto. Se argumenta que el modelo de las operaciones formales está demasiado centrado en el pensamiento adolescente y el pensamiento pre-adulto. Se considera, en consecuencia, que existen tipos de pensamiento que no muestran la estructura lógica de las operaciones formales o de estadios inferiores. Estos tipos de pensamiento podrían desarrollarse de manera paralela o después de las operaciones formales y podrían suplantarlas o sustituirlas, siendo utilizadas en las mismas áreas que las operaciones formales o en otras. En definitiva, lo que se apunta es que una comprensión adecuada del desarrollo del pensamiento no puede estar basada únicamente en el modelo de Piaget sobre las operaciones formales (Commoms et al., 1984: xv). Se considera así que las operaciones formales del pensamiento enfatizan el poder de la lógica pura en la solución de problemas. De este modo el pensador formal utiliza la lógica, el análisis racional, para buscar una correcta solución a un problema en función del dominio particular en el que ese problema está situado. Aún reconociendo que las operaciones formales del pensamiento proporcionan una poderosa y profunda perspectiva explicativa, se entiende que es insuficiente para comprender otras parcelas (no formales, "abstractas") como el mundo físico y social que, de esta manera, se han infravalorado en la teoría (Rybash, Hoyer y Roodin, 1986).

$3^{\circ}$. Del mismo modo, la perspectiva psicogenética ha desconsiderado los aspectos pragmáticos de la vida diaria. Se señala al respecto que el pensamiento operacional formal concede un gran énfasis a la posibilidad y la abstracción, y sin embargo, una escasa atención a aspectos que tienen que ver con el entramado de situaciones, vivencias, percepciones o estímulos propios de la vida cotidiana de cualquier individuo. La mayor parte del pensamiento formal tiene que ver con ideas, abstracciones y absolutos. De hecho, el razonador formal puede asumir equivocadamente que la meta del pensamiento maduro es construir un conjunto de principios puros y absolutos aplicables a los problemas de la vida diaria. En la última etapa de la adolescencia o pre-adultez, los individuos llegan a ser conscientes del excesivo énfasis en la abstracción y en la lógica, y encuentran difícil apli- 
cación de ésta a los problemas de la vida real (donde, en general, los problemas son sociales e interpersonales en naturaleza).

$4^{\underline{a}}$. En la misma línea que la anterior, frente al "sistema cerrado" en el que se encuentran los problemas lógico-matemáticos, con los que opera el pensamiento formal (Basseches, 1984; Koplowitz, 1984), con un número finito y conocible de variables y que tiene de ese modo una solución o varias, únicas y perfectamente definidas, se plantean "los problemas reales" que, en contraste, son "abiertos". Es decir, carecen de límites claros entre los elementos del problema y el contexto dentro del que ocurren. Rybash y otros (1986) señalan a este respecto que en la vida de cualquier persona, por ejemplo una mujer, puede existir un número infinito de variables que emergen desde un contexto concreto y unas circunstancias vitales determinadas que debe considerar a la hora de tomar una decisión cualquiera de la vida diaria (tener un hijo, casarse o hacerse torero). Dadas las diferentes variables potenciales que pueden presentarse, a partir de las cambiantes circunstancias de la vida (la cuestión de si, por ejemplo, esta mujer debería o no tener un hijo puede tomar muy diferentes significados personales, que influirán en la ultima decisión que adopte, que puede ser radicalmente distinta a la de otra mujer o persona ante el mismo problema o situación). De este modo, para estos autores, cuando examinamos algún problema real se comprueba que las variables que lo rodean son esencialmente indefinidas y dinámicas debido a que se encuentran en un contexto sociocultural.

En suma, se entiende que el pensamiento formal constituye parte de un sistema "cerrado" de problemas, mientras que los problemas de la vida real son frecuentemente problemas del tipo "abiertos-cerrados".

5․ Otra crítica planteada a esta perpectiva de análisis sobre la cognición adulta es que presta un notable énfasis al proceso de solución de problemas y escaso interés al proceso de descubrimiento de problemas, que representaría la habilidad de los adultos para plantearse cuestiones generales sobre sí mismos, sus vidas y los fenómenos que les rodean.

$6^{\circ}$. Por último, se alude a la inutilidad de las operaciones formales para ayudar a las personas a "conocerse a sí mismas". Debido a que el individuo es constreñido por el sistema de las operaciones formales desde el que razona, es incapaz de conocer la existencia de este sistema (metacognición). Piaget sugiere por ejemplo que el pensador formal no comprende la estructura del pensamiento lógico-matemático y que, de alguna manera, no es consciente de su autorreflexión.

El conjunto de estas insuficiencias detectadas en el modelo de pensamiento formal ha derivado en el desarrollo de propuestas alternativas sobre la cognición, especialmente en lo que se refiere a la edad adulta, algunas de las cuales se encuentran asociadas a perspectivas de más amplio alcance, como las del procesamiento de la información y la del ciclo vital, y otras, de menor extensión como pue- 
den ser el "modelo de encapsulación" propuesto por Rybash, Hoyer y Roodin (1986, 1994) dando forma, todo ello, a una línea de trabajo en favor de un quinto estadio del desarrollo cognitivo que emerge en la edad adulta y que ha sido denominado como estadio postformal o también estilos postformales de pensamiento.

\section{Más allá de Piaget: el pensamiento postformal}

Como ya hemos señalado anteriormente, existe un grupo de estudios que se han ocupado en defender la estructura básica de la teoría piagetiana desde el momento que ésta no pretendía otra cosa que estudiar el desarrollo intelectual y el pensamiento desde el nacimiento hasta la adultez temprana. Sin embargo, tal y como hemos apuntado, otros estudios han puesto en entredicho algunos postulados centrales de esta teoría, en especial, el modelo lógico de las operaciones formales en el que se basa el pensamiento adolescente ${ }^{8}$. Próximos a este grupo de autores, revisionistas de los planteamientos de la teoría psicogenética, están quienes entienden la necesidad de ir más allá del cuarto estadio piagetiano, poniendo en serias dudas que este estadio sea el último, el equilibrio cognitivo final ${ }^{9}$. En una línea más crítica se situan actualmente un grupo de investigadores que no sólo dudan del carácter central de la lógica abstracta y formal sino que achacan al modelo de Piaget serias deficiencias, en particular, la ausencia de factores o dimensiones básicas en la explicación del desarrollo cognitivo, muy especialmente, la variable contextual. Merece la pena destacar también aquí nuevos planteamientos que introducen aspectos de gran interés en el tema de la cognición adulta. Nos referimos concretamente a una línea de trabajo que podría considerarse dentro del marco de la denominada cognición social y que mantiene que los individuos comparten sus procesos cognitivos con otros individuos así como con otras herramientas y artefactos. Esto significa que el pensamiento está situado en un contexto particular de intenciones, compañeros y herramientas, es decir, está distribuido socialmente (Brown, y Campione, 1990; Rogoff, 1990; Resnick y Otros, 1991; Pea, 1992; Levine; Resnick, y Higgins, 1993; etc.). Más aún, se entiende que en el con-

8. Son muchos los autores que han puesto de manifiesto la insuficiencia e inconsistencia de la lógica formal. En apoyo de esta crítica se cita al propio Piaget quien antes de su muerte renunció a la lógica formal como modelo en favor de la lógica intencional, la "lógica de los significados" (Piaget, 1980). Vid. Broughton (1984: 397).

9. En el primer libro en el que se trata el desarrollo cognitivo adulto más allá de las operaciones formales de Piaget, editado por Commoms, Richards y Armon en 1984 con el expresivo título Beyond formal operations. Late Adolescent and adult cognitive development se da cuenta de un conjunto de investigaciones que apoyan diferentes aspectos críticos sobre las operaciones formales. En opinión de estos autores las críticas se han planteado desde diferentes ángulos: desde la lógica matemática (Powell, 1980), la lógica dialéctica (Riegel, 1973), la filosofía de la ciencia o el razonamiento metacognitivo-metasistemático (Stevens-Long, 1979; Lefebvre-Pinard, 1980; Fischer, 1980; Sinnott, 1981; Basseches, 1982; Commons, Richards y Kuhn, 1982; Sternberg y Downing, 1982; Commons y Richards, 1984; PascualLeone, 1984, etc.). Vid. Rybas, Hoyer y Roodin (1986). 
texto social de las nuevas tecnologías de la comunicación, este tipo de herramientas informáticas y telemáticas prolongan la inteligencia humana (Resnick, 1996: 195) permitiendo a la gente percibir y pensar de un modo que no se podría lograr sin su ayuda y, en suma, amplian la capacidad cognitiva adulta.

Sin ánimo de exhaustividad, señalamos a continuación algunos de los aspectos apuntados a partir de un breve análisis de las perspectiva del procesamiento de la información, del ciclo vital y de la teoría de la "encapsulación".

\subsection{La cognición adulta desde la perspectiva del procesamiento de la información}

El declive de la influencia de los planteamientos de Piaget y colaboradores y el alejamiento de las teorías estructurales coincidió hace unas décadas con la emergencia de esta perspectiva, particularmente en el área de la atención, la memoria, la inteligencia o la solución de problemas. Como se sabe, a través de una metodología basada en la analogía del ordenador se estudian determinados procesos y estrategias cognitivas, concebidas no como estructuras aisladas, sino en interacción y actuando como procesos básicos que sustentan a otros procesos más complejos como pueden ser el razonamiento, la resolución de problemas o la creatividad. A partir de esto se considera que el desarrollo cognitivo adulto resulta de los cambios en el modo como el individuo adquiere, almacena y responde a la información. En suma, la perspectiva del procesamiento de la información presta mayor atención a los procesos internos que a los resultados que se producen desde la captación del estímulo hasta la emisión de una respuesta inteligente dada; es decir, su objetivo fundamental se centra en trazar el flujo de la información a través del espacio y el tiempo a lo largo del sistema de procesamiento de información.

Clemente (1996) ha reconstruido algunos de los resultados más interesantes derivados de esta perspectiva en relación con la repercusión que algunos procesos y factores cognitivos tienen sobre el aprendizaje en las edades adulta y de la vejez. Vale la pena mencionar algunas de estas conclusiones por sus indudables implicaciones educativas:

- Algunos estudios muestran que la percepción juega un papel clave en el procesamiento y se ve afectada a lo largo del desarrollo adulto, implicando a las distintas modalidades sensoriales (Whitbourne y Weinstock, 1986). La impresión general es que se produce una pérdida de la capacidad del sistema nervioso central para procesar los estímulos en todos los sentidos, así como para integrar la información.

- Los estudios sobre procesamiento y resolución de problemas muestran claramente que los individuos de edades avanzadas presentan peores resultados ante las pruebas realizadas en situaciones experimentales que cuando se analizan los mismos procesos en situación de vida real.

- Respecto a la creatividad en la edad adulta y en la vejez, se señala que en general, quienes fueron productivos cuando eran jóvenes siguen siéndolo 
hasta alrededor de los setenta años (Poon, 1985), rechazándose la creencia de que la creatividad declina a lo largo de la vida adulta (sobre todo en aquellos casos en los que no existen problemas de salud importantes y se da una adecuada motivación).

- En relación con determinadas estrategias metacognitivas, se entiende que éstas se refieren tanto a aquellos elementos cognitivos que permiten el conocimiento de las dificultades de la tarea o de las estrategias que mejoran la memoria, a las habilidades propias de recuerdo, como al control que el sujeto tiene de esos procesos. Esto supone que además de conocer sus estategias cognitivas, las personas deben ser capaces de ponerlas en práctica de modo eficaz, teniendo en cuenta las variables del contexto (Clemente, 1996). Por poner un ejemplo, algunos estudios avalan la hipótesis de falta de selección y de control en la utilización de estrategias adecuadas en los individuos de edad avanzada (Lachman y cols., 1979); otros por su parte señalan el mayor desconocimiento de sí mismos que presentan estos sujetos respecto al propio funcionamiento de la memoria, o que son menos eficaces en el uso de imágenes visuales (Bruce y cols., 1982).

- Por último, un importante número de estudios situados en la órbita de esta perspectiva ha centrado su atención sobre la memoria y el aprendizaje. Un análisis breve sobre estos estudios conduce a algunas conclusiones de interés. Por un lado, las investigaciones realizadas sobre el aprendizaje muestran que éste es posible hasta edades avanzadas tanto cuando se trata de adquisición de nuevos contenidos, como de habilidades y procedimientos. Sin embargo, este aprendizaje es sensiblemente más lento, porque lo es el procesamiento que se efectúa de la información. En la misma línea, y respecto a la memoria (a la que desde esta perspectiva se ha prestado una extraordinaria atención) se ha estimado que su pérdida a medida que pasan los años responde fundamentalmente al deterioro en la capacidad de memoria y a la reducción en la velocidad en el procesamiento (Smith, 1980). La diferenciación en la memoria de procesos de codificación y procesos de recuperación de la información, permite observar que cuando la edad afecta al funcionamiento del sistema nervioso se traduce en una limitación fisiológico-funcional para ambos procesos (vid. Clemente, 1996). Desde otro punto de vista (el representado por la denominada Teoría de los Niveles de Procesamiento de autores como Craik y Tulving, 1975); Craik 1977; o Arenberg y Robertson-Tchabo, 1977; etc.), esto se explica porque se da un mayor deterioro del procesamiento ascendente, que puede verse compensado con el procesamiento descendente y además se entiende que los adultos mayores procesan la información a un nivel de procesamiento más superficial que los adultos más jóvenes. Algunos estudios señalan al respecto que el la mayor lentitud en el aprendizaje implica que los individuos mayores necesitan más tiempo. En consecuencia, si la adquisición de conocimiento con la edad es más lenta, es necesario practicar y ensayar 
más, lo que puede provocar fatiga, y en consecuencia en muchas ocasiones, se pierde en eficacia en la secuencia de enseñanza, favoreciendo el abandono de tareas o la falta de interés.

La perspectiva del procesamiento de la información no ha sido tampoco ajena a ciertas críticas. Una de las principales es la que señala la dificultad a la hora de operacionalizar algunos de sus supuestos más básicos. También se ha apuntado que los adultos resuelven problemas y procesan la información en función del significado que éstos tienen para su desarrollo personal (tesis mantenida por ejemplo por autores como Labouvie-Vief, 1977; Rybash y cols., 1986; Sternberg, 1986; etc.). Estos problemas además surgen en un contexto determinado que proporciona las coordenadas precisas para su resolución y que el individuo considera. Es decir, se trata de una idea que se aproxima notablemente a las posturas contextualistas en las que se enfatiza la importancia de la utilización de estrategias contextualizadas y pragmáticas ( $y$ no las abstractas y descontextualizadas que generalmente se dan en situaciones de laboratorio o en el frío ordenador).

\subsection{La cognición adulta desde la la perspectiva del ciclo vital}

En realidad, esta perspectiva resulta ser un armazon general para el estudio de la cognición y el desarrollo a lo largo del ciclo vital, sin embargo, puede apreciarse en ella aspectos distintivos sobre el desarrollo y la conducta cognitiva adulta (Alexaner, Druker y Langer, 1990; Baltes y Baltes, 1990; Featherman, Smith y Peterson, 1990; Labouvie-Vief y DeVoe, 1991). Rebok (1987: 16 y ss.) resume en tres las posiciones en las que se sitúa la perspectiva del ciclo vital respecto al desarrollo cognitivo adulto y en la vejez.

1. El desarrollo cognitivo es un proceso que cambia contínuamente (nuestro conocimiento cambia de manera continua desde la infancia hasta la vejez)

2. El desarrollo cognitivo implica una interacción de la persona y el contexto (existe una interacción dinámica entre el desarrollo personal y el cambio ambiental).

3. El desarrollo cognitivo es un proceso determinado por las características de multidireccionalidad y multiplicidad.

\subsubsection{El desarrollo cognitivo adulto como un proceso en continuo cambio}

- Se crítica a los modelos tradicionales que describen el cambio cognitivo como un proceso secuencial en constante evolución hasta un punto final máximo (situado al término de la adolescencia), a partir del cual se produce un período de estabilidad al que sigue la regresión y el declive, paralelo al declive físico o biológico. 
- Se afirma una notable confianza en el potencial de desarrollo cognitivo y se defiende la idea de cambios en cada período de edad del ciclo vital. Estos cambios además pueden ser de "avance y de retroceso". Es decir, pueden producirse regresiones en el desarrollo cognitivo (esta idea ya estaba presente en Vygotsky). No existe en consecuencia un estado final establecido y fijado a una edad determinada, sino que éste, de existir, es variable de unos individuos a otros. De esta manera, el crecimiento cognitivo y el declive puede ocurrir durante algún periodo del desarrollo-infancia, niñez, adolescencia, adultez o vejez (Labouvie-Vief, 1982). En este sentido, por ejemplo Rebok (1987: 16) señala que, del mismo modo que los niños muestran a veces regresiones cuando se trata de resolver un problema nuevo, también los adultos y los ancianos parecen regresar a niveles previos de funcionamiento cognitivo en variadas tareas, sobre todo en resolución de problemas lógicos. La cuestión está en que muchas de esas regresiones pueden deberse a otro tipo de variables o factores como una deficiente escolarización (en el caso de la mayoría de ancianos hoy esto es un hecho), problemas sociales o de salud y no necesariamente a declive cronológico.

\subsubsection{El desarrollo cognitivo adulto como una interacción de persona y contexto}

El aspecto sustantivo de todo este planteamiento es el especial énfasis puesto en la influencia que las variables contextuales, culturales y de interacción personal a lo largo de todo el ciclo vital (el ambiente familiar, los grupos de iguales, los espacios y contextos personales y sociales que cada individuo configura a lo largo de su vida) tienen sobre las posibilidades de crecimiento cognitivo. Como señala Rebok (1987) el enfoque de ciclo vital concede una gran importancia no sólo a los contextos inmediatos o próximos del desarrollo, sino también a los contextos pasados o distantes. Es decir, se presta una gran atención a los efectos generacionales y de coborte, decisivos tanto para el desarrollo cognitivo como para el psicosocial.

\subsubsection{El desarrollo cognitivo adulto como proceso multidireccional y múltiple}

Para la perspectiva contextualista, los cambios que acontecen a las personas a lo largo de sus vidas son definidos por las nociones de multidireccionalidad $y$ variabilidad (frente a nociones clásicas centradas en los cambios unidireccionales y acumulativos). Un ejemplo concreto de multidireccionalidad puede verse (para un mismo individuo) en el hecho que puede producirse un incremento de la parte de la inteligencia que tiene que ver con las destrezas verbales, en contraposición con el deterioro que sufren aquellos componentes de la misma que tienen un contenido ejecutivo. Por su parte, la variabilidad se explica por el carácter multicausal 
de los determinantes que influyen en el desarrollo cognitivo. De este modo, las funciones cognitivas adultas se ven influidas de manera diferencial de unas personas a otras en función de como sean asumidos y experienciados determinados eventos vitales no directamente relacionadas con la edad cronológica (promoción profesional, cambios familiares, enfermedades graves etc.).

\subsection{El modelo sobre la cognición adulta de Rybash, Hoyer y Roodin $(1986,1994)$}

El denominado modelo de encapsulación puede considerarse como una derivación de la línea de los estilos postformales de pensamiento. Este modelo trata de integrar, desarrollar y ofrecer una alternativa a algunos de los enfoques tradicionales en el estudio de la cognición, proponiéndose analizar los cambios relacionados con la edad en lo que considera son las tres ramas más importantes de la cognición adulta: procesamiento, conocimiento y pensamiento (Rybash, Hoyer y Rodin, 1986: p. IX), e interpretando la cognición a través de estas tres dimensiones interrelacionadas. De este modo los autores entienden que es posible integrar conocimientos de las tres líneas de investigación que más atención han dedicado a cada uno de esas dimensiones: psicométrica, epistemología genética y procesamiento de la información.

Para estos autores cada una de esas dimensiones ha sido estudiada erróneamente por las tres perspectivas citadas al considerarlas de manera independiente y aislada: la línea psicométrica ha centrado su atención sobre la inteligencia, ofreciendo una visión generalmente negativista sobre la adultez (en particular la adultez tardía) y más especialmente la vejez, según la cual el crecimiento evolutivo en las primeras etapas de la vida se convierten en estabilidad o involución en la adultez y vejez respectivamente. La epistemología genética se ha centrado sobre el pensamiento, pero cayendo en un notable reduccionismo a la hora de conceptualizar la cognición, centrada específicamente sobre el desarrollo del pensamiento lógicomatemático, subsumiendo en él otros dominios y otros tipos de pensamiento posibles. Por último, la teoría del procesamiento de la información, la inteligencia artificial y la ciencia cognitiva en general, focalizan su atención sobre las dimensiones del conocimiento adulto, en particular, el crecimiento y representación de los sistemas de conocimiento experto. En este caso, los resultados a los que llegan los investigadores de estos enfoques concluyen que los adultos son menos expertos en la solución de problemas generales y que existe una pérdida progresiva de información en el procesamiento.

Frente a esta idea, la teoría de la "encapsulación" estima que, muy al contrario, se da un incremento en el conocimiento adulto, pero sólo en unas áreas y no en otras, precisamente aquéllas que se desarrollan en forma "encapsulada". Se entiende que estas áreas, en tanto que estructuras de conocimiento autoconstruidas y cognitivamente accesibles al individuo, resultan de las habilidades generales, gracias a la experiencia personal (Rybash, Hoyer y Rodin 1986: 123). 
La teoría de la "encapsulación" parte del supuesto de que los adultos piensan sobre su conocimiento acumulado de una forma postformal, entendiendo por tal un número específico de estilos de pensamiento que son los que de alguna forma guían la conceptualización del conocimiento a nivel dinámico y activo. Dicho de otro modo, el desarrollo cognitivo adulto implica el crecimiento del conocimiento experto y la emergencia de estilos postformales de pensamiento, de manera que el resultado final de la "encapsulación" adulta es el estilo postformal del pensamiento y determinadas formas de conocimiento especializado dentro de campos o dominos específicos (Hoyer y Ribash, 1994: 10). Los autores sugieren que la acumulaciòn de conocimiento dentro de dominios substantivos juega un papel distintivo en el desarrollo adulto.

En resumen, ese proceso de "encapsulación" se entiende como una realización adaptativa, necesaria y constituye una característica distintiva del desarrollo cognitivo adulto frente a otras etapas anteriores o posteriores del ciclo vital. Es decir, la encapsulación del pensamiento y del conocimiento dentro de campos o áreas específicas (dominios específicos) representa una necesidad para afrontar las presiones y demandas sociogenéticas, de manera que los estilos de pensamiento y las formas de conocimiento que se derivan del encapsulamiento de los procesos cognitivos básicos ayudan a los adultos a adaptarse a problemas sociales y personales de la vida real y a limitar posibles factores estresantes. En consecuencia, los adultos ponen en relación estilos postformales de pensamiento con sus conocimientos acumulados en el dominio de conocimiento personal (individual y distintivo); de esa forma el conocimiento llega a estar "encapsulado" dentro de campos o áreas particulares y es puesto en ejecución cuando el contexto así lo demanda. Este último aspecto resulta de especial interés para la teoría y práctica educativa puesto que reitera la idea que la actividad cognitiva en la adultez está frecuentemente muy contextualizada, esto es, condicionada por el grado de estimulación/exigencia de los contextos en los que esa actividad tiene lugar (Hoyer y Ribas, 1994: 10).

\section{APROXIMACIÓN A LA CARACTERIZACIÓN DEL PENSAMIENTO POSTFORMAL: EL QUINTO ESTADIO DEL DESARROLLO COGNITIVO}

Como hemos señalado, son muchos los investigadores que, sobre todo desde principios de los años ochenta, están revisando y analizando la naturaleza del pensamiento adulto. Siguiendo a autores como Kramer (1983); Commons, Richards y Armon, (1984); Rybash, Hoyer y Roddin (1986); Arlin (1975a, 1984); etc.; podemos extraer de algunas de estas aportaciones sobre los estilos postformales del pensamiento adulto las siguientes características generales en las que coinciden la mayoría de ellos: 


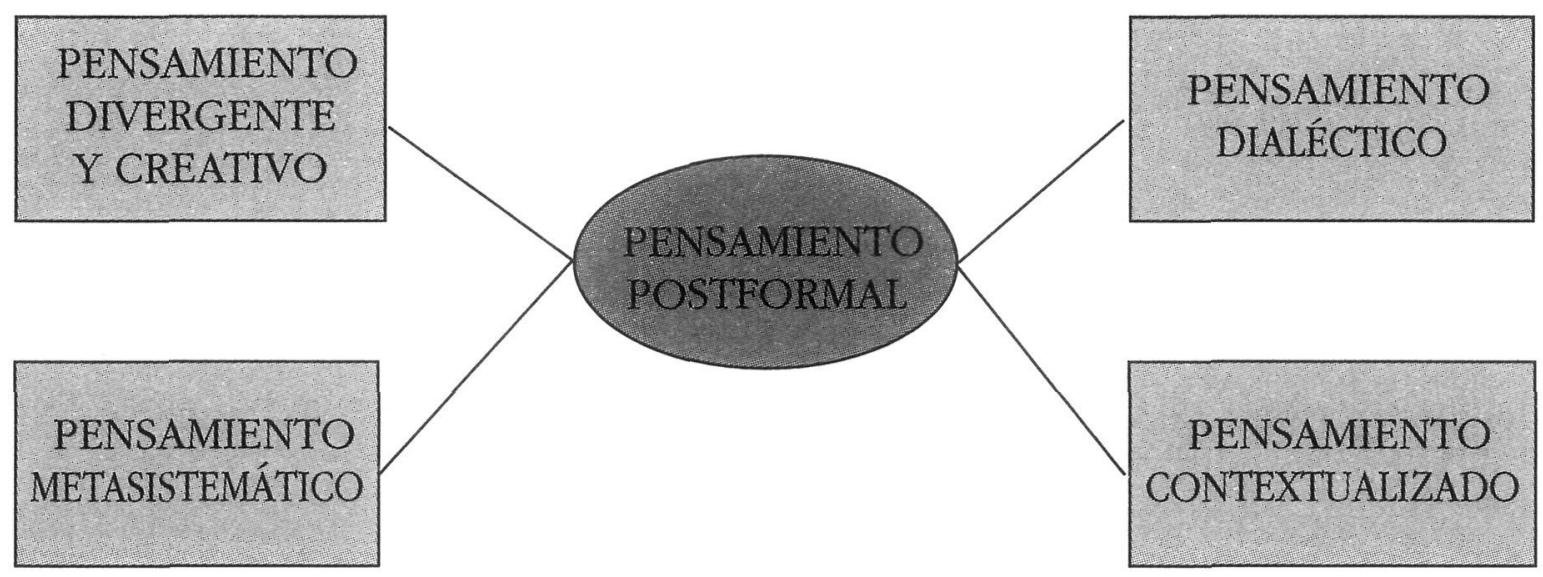

Características básicas del pensamiento adulto.

1. El pensamiento adulto es un pensamiento divergente. El pensador postformal acepta la contradicción como un aspecto básico de la realidad. Por ejemplo, en el plano de las relaciones personales pueden darse situaciones aparentemente contradictorias y simultáneas de amor y odio (las cosas no son radicalmente «blancas o negras") que no son excluyentes. Esto significa que la naturaleza del pensamiento es relativa y no absoluta. De este modo, el pensador postformal observa la realidad como algo temporal, y no como algo estático y fijado universalmente. Esta característica del pensamiento postformal adulto permite al individuo desarrollar la habilidad para sintetizar pensamientos, emociones o experiencias contradictorios dentro de un conjunto más amplio, integrado y coherente. Como señala Kramer (1983) esto explica el recurso que poseen las personas adultas para la integración de diferentes y "lógicamente" contradictorias alternativas y que no aparece, al menos no de modo tan claro, en el "pensador formal".

A principios de los años 50 Guildford en su modelo estructural de la inteligencia destacó la habilidad de "descubrimiento" y el pensamiento divergente (frente al pensamiento convergente) como dos de los rasgos cruciales para la cognición. Estudios posteriores sobre el pensamiento divergente y su relación con la edad cronológica han corroborado, mediante el uso de metodología longitudinal (McCrae, 1987; McCrae, Arenberg y Costa 1987; Simonto, 1991; Sasser-Coen, 1993), este tipo de resultados, destacando la presencia de rasgos como la flexibilidad, fluidez y originalidad como característicos del pensador divergente. Del mismo modo, algunos estudios como por ejemplo los realizados por Patricia Arlin (1975b, 1977, 1984) entienden que una característica básica del pensamiento postformal es que no sólo permite el desarrollo de procedimientos heurísticos para resolver determinadas situaciones y problemas, sino que además permite descubrir nuevos problemas, que generalmente no tienen una única solución, sino varias, en las que hay que considerar tanto elementos cognitivos, como emocionales, seleccionando aquéllas que sean más realistas y adaptadas al contexto. Para esta autora el descubrimiento de problemas es una habilidad muy relacionada con la originalidad, es 
decir, está muy relacionado con el pensamiento creativo. Se entiende por tanto que el descubrimiento de problemas puede ser una característica del desarrollo cognitivo postformal que es alcanzado por algunas personas creativas o inventivas, que desde luego han desarrollado las operaciones formales.

Por otro lado, aunque relacionado con lo anterior, autores como Dittmann-Kohli y Baltes (1986) sugieren que los adultos pueden desarrollar una serie de habilidades que coinciden con las que presenta el pensamiento postformal y que pueden ser integradas dentro del concepto de sabiduría, cuyas características básicas serían: a) la habilidad para utilizar la experiencia dentro del dominio del pensamiento personal; b) el énfasis en los aspectos pragmáticos o prácticos de la inteligencia y del conocimiento; c) el énfasis sobre el contexto del problema; d) la incertidumbre como una característica de problemas y soluciones; e) la reflexión y el relativismo necesarios en cualquier tipo de juicios y acciones.

2. El pensamiento adulto es un pensamiento metasistemático. A partir de este primer rasgo característico del pensamiento postformal, Rybash, Hoyer y Armon (1986) señalan que si un individuo puede observar la realidad en términos no sólo lógicos, sino relativos y contradictorios, entonces esta realidad (y el conocimiento de la misma) debe estar configurada como una estructura que adopta la forma de sistema abierto y dinámico, constituido por múltiples dimensiones que interactúan entre sí, lo que permite a los adultos contemplarla en su globalidad, de modo integral y completo. Pero además de esto, esta realidad está formada por múltiples sistemas, por tanto no es posible considerar uno único sino varios. Autores como Commons, Richards y Kuhn (1982), Armon (1984); Labouvie-Vief, 1982, 1984; Sinnott, 1981, 1984), etc. mantienen que el pensamiento formal comprende operaciones que se aplican sobre elementos de un sistema, o sistemas simples, en cambio el pensamiento postformal engloba operaciones que son aplicadas a múltiples sistemas de referencia. La cognición sobre diversos sistemas y sus interrelaciones son denominadas operaciones metasistemáticas por estos autores. La naturaleza del pensamiento postformal ha sido contemplado de este modo como intersistémico y metasistémico.

3. El pensamiento adulto es un pensamiento contextualizado. En el contexto de la vida diaria, el pensador postformal adulto no puede guiarse exclusivamente por principios universales, rígidos, lógicos y absolutos, dada la naturaleza en permanente cambio de la vida cotidiana y real. De este modo, el adulto crea nuevos principios basados en las circunstancias cambiantes y específicas de su vida (parafraseando a Ortega, se podría decir ' mis circunstancias y yo', sin las cuales el pensamiento adulto no se produce).

4. El pensamiento adulto es un pensamiento dialéctico. Uno de los principales críticos a la teoría de Piaget ha sido Klaus Riegel quien en 1976 en su "Manifiesto dialéctico " abogaba por abandonar y sustituir esta teoría basada en el equilibrio y la estabilidad por otro planteamiento basado en el desequilibrio y el 
cambio, apoyándose teóricamente para ello en el concepto filosófico de la dialéctica. En efecto, la idea del pensamiento dialéctico propuesto por primera vez por K. Riegel (1973), derivada de la noción y aplicación filosófica y del sociologismo marxista (Hegel, Marx y sus seguidores), y empleada también en la física o en las matemáticas, es utilizada tanto al desarrollo psicosocial como al desarrollo cognitivo adulto, en donde pone de relieve la existencia de un conflicto permanente entre fuerzas interactivas en continuo cambio. También aquí se enfatiza el conflicto entre una idea (tesis) y su opuesta (antítesis). La resolución del conflicto (síntesis) tiene un carácter temporal debido a que las fuerzas interactuantes son constantemente cambiantes. Existe un cierto consenso en considerar que en este tipo de pensamiento dialéctico se combinan cuatro dimensiones del desarrollo: una de carácter individual-psicológica; otra de tipo individual-biológica; la tercera de tipo cultural-sociológica y otra dimensión que abarca variables de tipo físico.

Después de las primeras ideas de Riegel, ha sido sobre todo Michael Basseches $(1980,1984)$ quien más ha trabajado la idea del pensamiento dialéctico, aunque con un planteamiento diferente al de Riegel $(1973)^{10}$ dando forma a una importante línea de investigación sobre el pensamiento postformal caracterizado, de este modo, en los términos de pensamiento dialéctico. Para Basseches (1986) este tipo de pensamiento va más allá de las limitadas condiciones del análisis formal, y puede ser definido como una forma de organización cognitiva que parece presentarse en algunos individuos durante los primeros años de la adultez y que genera en el pensamiento un nuevo nivel de equilibrio que trasciende las estructuras cognitivas piagetianas (para Basseches -1986: 48- "la dialéctica es desarrollo en transformación". Se refiere a la transformación evolutiva de sistemas a lo largo del tiempo, via relaciones constitutivas e interactivas). La idea básica que subyace aquí es que el pensador postformal percibe la realidad como un conjunto estructurado de formas y sistemas en evolución, transformación y cambio continuo. Esos cambios, que tienen lugar en un mundo a su vez cambiante, son integrados por las personas gracias a los principios de la dialéctica, mediante relaciones constitutivas e interactivas entre el todo y las partes, dentro de sistemas múltiples y abiertos (Basseches, 1984).

4. DisCuSIÓN: La COGNICIÓN ADULTA, IMPLICACIONES PARA LA EDUCACIÓN EN LA ADULTEZ Y VEJEZ

Iniciábamos este trabajo haciéndonos eco del amplio reconocimiento a la teoría de Piaget; sin embargo, como señala Miras (1996), en las propias virtualidades de la teoría genética se encuentran sus limitaciones. Una en la que hemos insistido

10. Riegel describió el pensamiento dialéctico en un modo que no presupone el pensamiento formal. En cambio Basseches lo describe dentro de una amplia red o armazón que integra el pensamiento operacional formal. 
aquí es que frente a la etapa de las operaciones formales, caracterizada como cognitiva, convergente, de lógica formal, proposicional, estructural, abstracta, descontextualizada, general, transindividual, absoluta, universalista, dicotomizada, monológica (más que dialógica) y empírica (más que transcendental) (Broughton, 1984: 400) puede entreverse un nuevo estadio, el quinto estadio del desarrollo cognitivo humano, que supera algunas de las limitaciones del anterior y que surge cuando el adulto acomoda el sistema operacional formal, lógico, a la realidad de la vida diaria. Cuando sucede esto, el adulto encuentra varias realidades mutuamente contradictorias que coexisten confortablemente. Este estadio coincide con la adquisición de conceptos nucleares como son los de relatividad (Sinnott, 1984) ${ }^{11}$ o dialéctica. De este modo, y dada la posición central de estas características, a este quinto estadio se le denomina también estadio dialéctico o relativo, que como hemos dicho permite que los adultos resuelvan problemas que se producen en escenearios de la vida real, característica ésta en la que insistiremos más adelante.

El amplio acuerdo existente entre los diferentes grupos de investigadores en reconocer la presencia de un nuevo estadio cognitivo permite empezar a pensar que se trata de un nivel cualitativamente diferente al de las operaciones formales descrito por Piaget, y no una parte de éste como también se ha defendido. Algunos autores como Koplowitz, Labouvie-Vief, Basseches, Powell, Arlin y otros, etc. coinciden en la presencia de las características del pensamiento adulto señaladas anteriormente y que podrían resumirse, con Kramer (1983), en las siguientes: $1^{\circ}$ ) la aceptación y el reconocimiento de que el conocimiento es relativo y no absoluto; $2^{\circ}$ ) la aceptación de la contradicción entre diferentes puntos de vista; $3^{\circ}$ ) la integración o síntesis de las contradicciones en un sistema más inclusivo; y $4^{\circ}$ ) pragmatismo.

Sin embargo, también hay otros autores (Rybash, Hoyer y Roodin, 1986; Hoyer y Rybas, 1994) que prefieren hablar, en vez de una nueva etapa cognitiva, de un conjunto de estilos postformales de pensamiento, que emergen durante la adultez. Entre los 'estilos' más importantes se incluirían la capadidad de descubrir problemas, el pensamiento relativo (investigado mediante el uso de dilemas sociales) y sobre todo el pensamiento dialéctico, sobre el que han mostrado interés un importante número de investigadores, especialmente Arlin (1977); Sinnott y Guttmann (1978; Smith (1978); Basseches (1980); Fowler (1981); Edelstein y Noam (1982); Kegan (1982); Pascual-Leone (1984); etc.

Sea como fuere, lo cierto es que el análisis dialéctico o la dialéctica es considerado por una y otra postura como el principio organizador del pensamiento ya que ofrece un nivel de equilibrio distinto al principio organizador del pensamiento de las operaciones formales que es el conjunto estructurado o sistema. De este

11. Vid. Sinnott, D. J. (1984) En Commoms y otros (eds.). Op. cit. Cap. 14, pp. 309 y ss. Para Sinnot las operaciones relativas proporcionan a los adultos la posibilidad de ampliar su márgen de elecciones y de mejorar sus interpretaciones sobre la realidad social, haciéndolas más flexibles e inteligentes, con lo que se entiende que el pensamiento relativo tiene un impacto sobre la conducta del individuo y sobre el grupo social (Sinnot, 1984: 325). 
modo, el pensamiento dialéctico se sitúa dentro de una forma particular de organización del pensamiento que es el nivel metasistemático de organización cognitiva. Es decir, aparece como un proceso de transformación del desarrollo que se constituye en una via de relaciones interactivas a través de las cuales los sistemas evolucionan y cambian (Basseches, 1984: 236). Sin embargo, no todos los jóvenes adultos alcanzan este nivel de pensamiento, del mismo modo que no todos los adolescentes alcanzan el nivel de las operaciones formales. La pregunta aquí surge de inmediato, entonces ¿qué factores condicionan que se alcance el nivel del pensamiento dialéctico y con ello el equilibrio cognitivo? Algunos investigadores se han atrevido a apuntar algunas respuestas. Por ejemplo, Basseches (1986: 53) señala que la respuesta a esta cuestión es doble. Por un lado, los factores ambientales (fundamentalmente para él, las relaciones interpersonales) y la actividad educativa orientada a la práctica y a la resolución de problemas de la vida real.

Considerando la primera de estas cuestiones, el llamativo reduccionismo en el que cae la teoría genética al considerar el desarrollo humano jalonado por estructuras cognitivas y mecanismos funcionales comunes y universales para todos los sujetos supone, entre otras cosas, negar la importancia de los efectos contextuales y especialmente las diferencias culturales en el proceso del desarrollo cognitivo. Implica no considerar suficientemente la interrelación entre diversos elementos como son el contexto, las fuentes de identidad, los guiones de vida personalizados (Hendricks, 1999) y que son enfatizados por planteamientos que se sitúan en la línea de Vygotski y del modelo relacional o contextual (lifespan).

Precisamente, la importancia de las diferencias individuales en la cognición, además de las diferencias contextuales y culturales, se acentúan más, si cabe, en el período adulto y, en gran medida, ha favorecido que la investigación cognitiva haya empezado a salir del laboratorio y a interesarse por entornos de aprendizaje más naturales, como puedan ser el lugar de trabajo, diferentes espacios de intercambio sociocultural, de educación informal, contextos sociales en los que es necesario el uso de las nuevas tecnologías de la comunicación, y en general, los escenarios de la vida cotidiana (Brown y otros, 1989; Pea, 1992, etc.), todo ello sobre la base según la cual se considera que la cognición adulta es un proceso socialmente compartido y distribuido en el plano de la vida diaria.

La idea de que un aspecto distintivo del pensamiento adulto es su aplicación a la esfera de la vida real y cotidiana ya estaba presente en la teoría sobre el desarrollo adulto propuesta por Schaie a finales de los años $70^{12}$. Sin embargo, hoy día

12. En esa teoría Shaie y cols. señalan que en las primeras etapas de la infancia y adolescencia se adquiren las habilidades y destrezas intelectuales y cognitivas necesarias; en la adultez temprana es necesario aplicarlas en el mundo laboral o de la formación; en la adultez media el individuo debe responsablemente ejecutar estas habilidades cognitivas dentro un marco normalizado de vida diaria, tanto del ámbito familiar, como profesional o comunitario; por último, en la fase de la vejez, los individuos se ven más influenciados por variables de tipo actitudinal o motivacional, y donde la "disposición mental" del individuo ante determinados problemas tiene mucho que ver con el grado de significación personal que éstos tengan para él. 
toma nuevas formas. Por ejemplo, una de las característica más interesantes del modelo de encapsulación, al que aludíamos más arriba, es que enfatiza el hecho de que frecuentemente la actividad cognitiva en la adultez está contextualmente basada. Como apuntan Craik y Jennings (1992) el pensamiento, el razonamiento y otros aspectos del funcionamiento cognitivo diario en la adultez no están más condicionados por la epigénesis que lo que puedan estar por efecto de los contextos en los cuales esas actividades tienen lugar. Por ejemplo, han argumentado que los declives relacionados con la edad en la función de la memoria son debidos a las dificultades de recuperación y codificación en ausencia de contextos de apoyo. En suma, algunos estudios han mostrado cómo la varianza asociada con la interacción entre los procesos de memoria y los contextos en los que operan llegan a ser más significativos que los efectos del envejecimiento (Park y Shaw, 1992).

Una apreciación necesaria en este punto es que no sólo el contexto, tomado en sentido general, sino particularmente los contenidos de aprendizaje son fundamentales para el desarrollo cognitivo. Dicho con otras palabras, el pensamiento, la inteligencia, o el aprendizaje dependen del tipo de conocimiento; es decir, que lo que el individuo es capaz de conocer, de construir como nuevo conocimiento, o lo que hace que alguien sea inteligente depede en gran medida de lo que ya sabe (Ackerman, 1996). En el caso de los adultos, especialmente cuando éstos se encuentran alejados del medio escolar o académico, el conocimiento adquirido de manera informal generalmente no es utilizado por el medio escolar, desaprovechándose una interesante fuente de conocimiento para favorecer la continuidad del aprendizaje (Resnick, 1996: 191). Por otro lado, el interés en la temática de los contenidos se refleja en la perspectiva de la cognición específica de dominio. Los trabajos que se sitúan en esta perspectiva comparten la idea de que la cognición puede variar sustancialmente según las diferentes áreas o tipos de contenidos y tratan de fundamentar teóricamente y encontrar evidencias de la existencia de sistemas de cognición específicos a determinados dominios (Miras, 1996: 48). Dentro de esta perspectiva, aquí hemos analizado de manera más particular el modelo de autores como Rybas, Hoyer; Hoyer y Rybas (1994); Wellman y Gelman, (1992) etc., para los que la encapsulaciòn es una característica propia de la adultez. Como hemos señalado, este modelo específicamente sugiere que la cogniciòn adulta es distintivamente asíncrónica o individualizada, elástica y encapsulada, en relaciòn con etapas anteriores o posteriores de la vida, características que tomadas conjuntamente porporcionan una base para un paradigma del desarrollo cognitivo adulto (Hoyer y Rybash, 1994: 8) en el que se destaca idea de la diversidad y de variabilidad interindividual entre los adultos. La teoría señala que el conocimiento adulto llega a ser encapsulado y específico durante este periodo del ciclo vital, en el incremento de ese conocimiento incide de forma especial la experiencia, la automatización, la intuición, la autoconstrucción y la propia actividad del sujeto. De aquí se deriva la hipótesis fundamental de la teoría y es el hecho que las babilidades fundamentales de los adultos y ancianos continúan normalmente en función del dominio que bayan adquirido, es decir, que existen dominios del conocimiento dentro 
de los cuales los adultos no evidencian declive o no reducen su eficacia de función hasta bien avanzada la edad. Aunque la adquisición de nuevo conocimiento (el conocimiento no relacionado con lo ya encapsulado en específicos dominios) puede llegar a ser cada vez menos eficiente con el avance de la edad, sin embargo, la reducción de la capacidad para adquirir nuevo conocimiento puede ser compensada por el desarrollo de conocimiento experto dentro de los dominios existentes.

Trasladado el análisis anterior a la esfera de la reflexión e intervención pedagógica, conduce a pensar que si se acepta la importancia de los factores contextuales sobre el desarrollo cognitivo, es necesario asumir la trascedencia de facilitar y potenciar espacios y ambientes sociales de aprendizaje continuos, de modo que el individuo pueda mantener, desarrollar o ampliar sus esquemas de conocimiento, en interacción con el medio social e interpersonal. Por otro lado, se refuerza también la necesidad de orientar la práctica educativa hacia aspectos vitales y de interés para los adultos, de modo que permitan tanto apoyar el potencial cognitivo de éstos como evitar los efectos negativos que son consecuencia del desuso y del declive cognitivo asociado a los procesos de envejecimiento. Al respecto, en un trabajo reciente, Spinger-Littles y Anderson (1999) han comprobado cómo en la práctica educativa cotidiana con personas mayores este tipo de alumnos rinden mejor cuando se encuentran en un ambiente de aprendizaje que pueden controlar. Ese "Control" del ambiente para generar un adecuado aprendizaje puede favorecerse a través de recursos diversos. Por ejemplo en línea con lo dicho anteriormente, se entiende que para los alumnos adultos o mayores (en general para cualquier alumno), el aprendizaje es más eficaz cuando la nueva información se conecta con el conocimiento previo construido por el sujeto en el marco de sus experiencias de la vida real. Puesto que parece probado que los alumnos de edades avanzadas se encuentran bastante condicionados por sus creencias, conocimientos, valores y visiones sobre el mundo, desarrollados a lo largo de toda su vida, esto hace que experimenten un tipo de interferencia proactiva (Davis y Palladino, 1997) que aconseja un tipo de aprendizaje construido, no transmitido (pinger-Littles y Anderson (1999: 206), en el marco de un tipo de ambiente en el cual el instructor y los estudiantes entre sí proporcionan y comparten información. De este modo, se consigue conectar la nueva información con el conocimiento que ya poseen y con sus experiencias vitales. Por otro lado, los adultos y las personas mayores responden mejor en un ambiente de aprendizaje colaborativo (cooperativo), en el que se refuerzan las ideas de aprendizaje interactivo, mediado, que permita la interiorización de determinadas estrategias cognitivas en un contexto de interacción personal, para lo que también juega un importante papel el desarrollo de habilidades sociales.

En resumen, el aprendizaje con este tipo de alumnos es más efectivo primero, cuando la información es relevante en línea con sus problemas e intereses vitales y además el individuo comprende el porqué y cómo utilizar la nueva información. En segundo lugar, cuando se sustituyen viejas fórmulas pedagógicas por nuevas estrategias como pueden ser el aprendizaje autodirigido, la autoevaluación, el pensamiento crítico, los grupos de aprendizaje y técnicas para la búsqueda y acceso a 
la información, el aprendizaje guiado mendiante estilos de cuestionamiento, estrategias de aprendizaje cooperativo y grupos de discusión, etc.

El análisis anterior nos lleva a reforzar la idea de la importancia que tiene una adecuada intervención sobre los procesos sociales a lo largo del desarrollo adulto. El objetivo educativo de esa intervención es la de evitar un mayor deterioro asociado al declive biológico, y que en ocasiones acontece simplemente por la notable reducción de estimulación ambiental a la que los adultos se ven progresivamente expuestos. La importancia de una acción educativa contextualizada en las etapas de la adultez y vejez, en el fondo, remite a dos cuestiones diferentes, aunque notablemente relacionadas entre sí y que tienen un gran interés pedagógico, puesto que sin su resolución teórica y empírica, dificilmente se puede justificar la praxis educativa en estos dos tramos de edad. Nos referimos concretamente a las ideas de plasticidad cognitiva individual y de declive cognitivo.

En relación con la primera cuestión, hasta hace relativamente poco tiempo, la idea de plasticidad fue más fácilmente reconocida en el periodo de los primeros años de la vida, y no como una característica que se pudiera encontrar entre las personas adultas y mayores, suponiéndose que la plasticidad cognitiva o conductual era inversamente proporcional a la edad. Sin embargo, como apuntábamos más arriba, del mismo modo que para el desarrollo psicosocial, también aquí se puede hablar de fases y posibilidades de progreso y retroceso, de estancamiento y de cambio en un movimiento dialéctico. Como concluyen muchos investigadores sobre este asunto, los procesos y principios del cambio psicológico humano son universales, pero las particularidades de cada individuo a los que se aplica son únicas, y por lo tanto cualquier generalización resultaría inexacta. Para nosotros, esto refuerza la necesidad de promover recursos que favorezcan la posibilidad potencial de modificación y mejora de determinadas habilidades intelectuales en cada una de las personas, tomadas de manera diferencial.

El segundo aspecto, el análisis de los factores relacionados con el declive cognitivo, resulta notablemente interesante también, especialmente cuando se observan las contradicciones y la falta de consenso existente hoy día sobre el tema. De un lado, algunos investigadores, como por ejemplo Labouvie-Vief y Chandler (1978); Schaie (1979); etc., señalan que el declive cognitivo relacionado con el paso del tiempo no es apreciable hasta una edad cercana a los sesenta años; por el contrario, otros estudios siguen manteniendo las tesis de trabajos clásicos como los de Welford (1969), y defienden que el declive es continuo empezando a mediados de la segunda década de la vida. En cualquiera de los supuestos, la intuición práctica, el conocimiento que proporciona la experiencia (en el terreno personal o profesional) con personas mayores y, desde luego, la investigación, llevan inevitablemente a aceptar que efectivamente el declive cognitivo se produce en algún momento del proceso de envejecimiento (Salthouse, 1989). Lo que no está tan claro es si el declive se produce en todos los tipos de cognición o en todas las funciones cognitivas y tampoco qué factores son los responsables de este declive observado empíricamente. 
En el fondo, lo que queremos enfatizar con esto es que, tal y como señalábamos al principio de nuestro trabajo, no es posible considerar globalmente los diferentes tipos de funciones cognitivas y concluir que el declive cognitivo se da de igual modo para todas ellas. De hecho, si se analizan algunas de las funciones cognitivas más estudiadas en relación con este tema, como por ejemplo la inteligencia, la memoria o incluso la creatividad, se observa que existen diferentes hipótesis sobre las que han trabajado los especialistas durante mucho tiempo, algunas de las cuales optan, por un lado, por distinguir o parcializar en cada una de esas funciones, elementos, tipos o subfunciones y, por otro, en establecer relaciones con otras funciones o elementos cognitivos, de manera que esta parcialización y asociaciación permita explicar resultados empíricos aparentemente contradictorios entre sí.

Pongamos algunos ejemplos. En el caso de la inteligencia puede fácilmente observarse cómo distinciones clásicas como las realizadas por Hebb en los años. cuarenta $^{13}$, o la diferenciación hecha por Horn y Cattell entre inteligencia fluida e inteligencia cristalizada, permiten apreciar efectos cronológicos desiguales en uno u otro de estos tipos en función de diversos factores (por ejemplo, los procesos de influencia cultural y educativa o el deterioro del SNC). Por otro lado, representaciones teóricas recientes sobre la inteligencia adulta como la formulada por Ackerman (1996) proponen integrar las relaciones existentes entre personalidad, intereses, desarrollo del conocimiento y destrezas intelectivas (Ackerman, 1996: 237), dentro de un esquema explicativo en el que se conjugan los dos dos grandes aspectos de la inteligencia, tradicionalmente separados en la literatura especializada, como son la inteligencia como proceso y la inteligencia como conocimiento. Lo mismo podría aplicarse para la memoria ${ }^{14}$ o para la creatividad. En el caso de la memoria resulta innegable el declive relacionado con la edad encontrado en numerosos estudios (Salthouse 1991, Kausler, 1994; light, 1996; etc.), sin embargo los investigadores no se ponen de acuerdo sobre las causas o factores que explican el mismo. Como señala Kastenbaum (1992) para el caso de la creatividad, lo importante no es sólo saber que se produce un declive con el paso de los años, o cuándo ocurre y la magnitud del decremento, sino por qué ocurre ese decremento y a qué tipo de individuos afecta. En este último caso, algunos estudios afirman que de producirse ese decremento, es menos acusado en persona con adecuados niveles de salud, y con un buen nivel educativo; en otros, se ve relacionada con la inteligencia, la ejecución cognitiva, y con caractrísticas de personalidad (Hendricks, 1999).

En resumidas cuentas, resulta necesario considerar la complejidad del fenómeno cognitivo en su conjunto, y la dificultad de atender una única perspectiva

13. Como se sabe, Hebb distinguió dos tipos de inteligencia: tipo A y tipo B (la Inteligencia tipo A sería la habilidad para desarrollar nuevos patrones de respuestas y la Inteligencia tipo B sería el funcionamiento de estos patrones ya desarrollados). Para $\mathrm{Hebb}$, los procesos tipo A declinan con la edad, mientras que el tipo B continuan aumentando a lo largo de la adultez avazada).

14. Una interesante revisión de las diferentes hipótesis explicativas sobre las diferencias encontradas entre jóvenes y viejos en relación con el funcionamiento de la memoria operativa puede encontrarse en ELOSUA, R. Ma y LECHUGA, M․ T. (1999). Diferencias relacionadas con la edad en el funcionamiento de la memoria operativa. Cognitiva, vol, 11, 1, 109-125. 
explicativa o de análisis. Por ejemplo, siguiendo a Salthouse (1989), un análisis somero sobre el tema del declive congnitivo permite ver la existencia de diferentes hipótesis explicativas (desde las primeras hipótesis de la velocidad -en las que se explicaba el declive cognitivo debido a la reducción en la velocidad de los procesos sensorio-periféricos o motóricos-, la hipótesis del desuso-la falta de uso de determinadas funciones cognitivas, tal vez como consecuencia de la pérdida de estimulación procedente del medio: jubilación laboral, cese de determinadas actividades, menores responsabilidades familiares..., debilita y dificulta el recurso y la activación de esas funciones-; la hipótesis del ambiente cambiante-que sugiere que no es el individuo el que cambia sino lo que sucede es que los aspectos del ambiente físico o social están constantemente cambiando, lo que hace que las sucesivas generaciones de personas ejecuten progresivamente mejor que las anteriores los mismos tipos de tests-; etc.). En todas ellas, lo que se pone de manifiesto es que lo importante no es el declive relacionado con la edad sino los aspectos ambientales que, en último término, son responsables de los peores niveles de ejecución en las pruebas de medición. De igual modo, al analizarse otras hipótesis más actuales que consideran que ninguna de las tesis anteriores, por sí solas, sirve para explicar el fenómeno del declive cognitivo, se concluye que los resultados empíricos de los que se han extraído estas hipótesis no son concluyentes debido a que intervienen también otros factores explicativos como por ejemplo dificultades a la hora de responder rápidamente a los tests o de percibir y codificar las preguntas. Por otro lado, se sabe también que muchos de los tests aplicados fueron diseñados para individuos familiarizados con tareas escolares, con lo que tienen mucho más significado para individiduos jóvenes, permitiéndoles ejercitar mucho mejor las habilidades que se miden en esos tests que a los individuos adultos o mayores. Tal vez por ello, algunos estudios recientes van en la línea de destacar tareas específicas de procesamiento (componentes o funciones más específicas) y, por supuesto, en destacar también el efecto de otras variables como puede ser el estado de salud sobre el declive cognitivo.

A pesar de la dificultad del tema y más allá de trabajos de carácter puntual sobre la cognición en general o, más particularmente, interesantes aportaciones recientes sobre la pedagogía cognitiva (Vázquez Gómez y Bárcena, 1999), dada la escasa presencia de estudios sobre la cognición adulta en nuestra comunidad pedagógica, quisieramos terminar insistiendo en el punto que nos animaba al principio del texto que no es otro que intentar defender la idea de prestar una mayor atención al funcionamiento y desarrollo cognitivo, imprescindible para la teoría y práctica educativa con personas adultas y mayores. Entendemos que esto es así fundamentalmente porque sólo después de ese conocimiento es posible justificar, con razones basadas en el estudio científico y más allá de voluntarismos y paternalismos mejor o peor disimulados, la aplicación de intervenciones pedagógicas que permitan tanto mitigar los efectos del declive biológico, o retrasar los derivados del deterioro, como potenciar determinadas funciones cognitivas durante este tramo del ciclo vital. 


\section{BIBLIOGRAFÍA}

ACKERMAN, L. P. H. (1996a): A theory of adult intellectual development: process, personality, interests, and knowledge. Intelligence, 22, 227-257.

- (1996b): Intelligence as process and knowledge: an integration for adult developmente and apllication, en ROGER, A. W.; FISK, D. A.; WALKER, N. (eds.). Aging and skilled performance: Advances in theory and applications. Hillsdale, NJ.: Erlbaum, pp. 139-156.

ALEXANER, C. N.; DRUKER, S. M. y LANGER, E. J. (1990): Introductions: Mayor issues in the exploration of adult growth, en ALEXANDER, C.N. y LANGER, E. J. (Eds.): Higher stages of buman development: perspectives on adult growth. Oxford University Press. New York. pp. 3-32.

ARENBERG, D. (1973): "Cognition and aging: verbal learning, problem solving and memory" en C. Eisdorfer y LAWTON, M. P. (eds.) (1973): The Psichology of adult development and aging. American Psychological Associatioin. Washington, D.C.

ARENBERG, D. Y THOMPSON, L. (eds.) (1980): New directions in memory and aging. Hillsdale, NJ, Erlbaum.

ARLIN, P. K. (1975a): A cognitive process model of problem finding. Educational Horizons, 54 (1975-76), pp. 99-106.

- (1975b): Cognitive development in adulthood: a fifth stage?, Developmental Psychology, 11, pp. 602-606.

- (1977): Piagetian operations in problem finding. Developmental Psychology,13, pp. 247-298.

- (1984): "Adolescent and adult thought: a structural interpretation", en COMMOMS, M. L.; RICHARDS, F. A; ARMON, Ch. (eds.) (1984): Beyond formal operations. Late Adolescent and adult cognitive development. Praeger Publishers. New York. pp. 259-270.

- (1986): Problem finding and young Adult Cognition. En MINES, A. R. y KITCHENER, S.K. (1986): Adult cognitive development. Methods and models. Praeger Publishers. New York. pp. 22-32.

BALTES, B. P. y BALTES, M. M. (1990): Psicological perspectives on succesful aging: the model of selective optimization with compensation. En BALTES, B. P. y BALTES, M. M. (eds.) Succesful aging. Cambridge University Press. New York. pp. 1-34.

BASSECHES, M. (1980): Dialectical schemata: a framework for the empirical study of the development of dialectical thinking. Human Devleopment, 23, pp. 400-421.

- (1984): Dialectical thinking. Norwood, NJ: Ablex.

- (1984): Dialectial thinking as a metasystematic form of cognitive organization, en COMMOMS, M. L.; RICHARDS, F. A; ARMON, Ch. (eds.) (1984): Beyond formal operations. Late Adolescent and adult cognitive development. Praeger Publishers. New York. pp. 216-238.

- (1986): Dialectical thinking and young adult cognitive development. En MINES, A. R. y KITCHENER, S. K. (1986): Adult cognitive development. Methods and models. Praeger Publishers. New York. pp. 33-56.

BROUGHTON, M.J. (1984): "Not Beyond formal operations but beyond Piaget". COMMOMS,

M. L.; RICHARDS, F. A.; ARMON, Ch. (eds.) (1984): Beyond formal operations. Late Adolescent and adult cognitive development. Praeger Publishers. New York. pp. 395411.

BROWN, A. L. y CAMPIONE, J. C. (1990): Communities of learning and thinking,or a context by any other name. Contributions to Human Development, 21, pp. 108-126. 
BRUCE y cols. (1982): Adult age differences in metamemory. Journal of Gerontology, 37, pp. 354-357.

CLEMENTE, A. (1996): Psicología del Desarrollo Adulto. Narcea. Madrid

COLL, C. (1996): El legado de Jean Piaget (1896-1996). En Anuario de Psicología, no 6, 213220.

COMMOMS, M. L.; RICHARDS, F. A.; ARMON, Ch. (eds.) (1984): Beyond formal operations. Late Adolescent and adult cognitive development. Praeger Publishers. New York.

COMMONS, M. L., RICHARDS, F. A. y KUHN, D. (1982): Systematic and metasystematic reasoning: a case for levels of reasoning beyond Piaget's stage of formal operations. Cbild Development, 53, pp. 1058-1068.

CORNELIUS, S.W. (1990): Aging and everyday cognitive abilities. En HESS, M. T. Aging and cognition: Knowldge organization and utilization. Elsevier, Amsterdam.

CRAIK, F. I. M. (1977): Age differences in human memory. En BIRREN, E. J. y SCHAIE, W. K. (eds.). (1977): Handbook of psychology of aging. Van Nostrand Reinhold. N. York.

- y TULVING, E. (1975): Depth of processing and the retencion of words in episodic memory, Journal of Experimental Psychology: General, 204. pp. 268-294.

- y TREHUB, S. (1982): Aging and cognitive processes. Advances in the study of communication and affect. vol 8. Plenum Press. New York.

- y JENNINGS, J. M. (1992): Human memory. En CRAIK, F. I. y SALTHOUSE, T. A. (Eds.) Handbook of cognition and aging. Hillsdale, NJ: Lawrence Erlbaum Associates. pp. 51110.

DAVID, J. M.; KRIVINE, J .P y SIMMONS, R. (1993)(eds.) Second Generation expert Systems. Springer. Berlin.

DIXON, R.A. (1989): Questionaire research on metamemory and aging: Issues of structure and function, en L. W. POO, D. C. RUBIN y B. A. WILSON (eds.) Eveyday cognition in adultood and later life. Cambridge University Press, Cambridge. pp. 394-415.

EDELSTEIN, W. y NOAM, G. (1982): Regulatory structures of the self and "postformal" stages in adulthood. Human Development, 25. pp. 407-422.

EYSENCK, H. J. (1995a) Creativity as a product of intelligence and personality. En D. H. Saklofske Y ZEIDNER, M. (Eds.) International bandbook of personality and intelli.

- (1995b): Genius: The natural bistory of creativity. Cambridge University Press. New York.

FEATHERMAN, D. L.; SMITH, J. y PETERSON, J. C. (1990): Successful aging in a post-retired society. En. En BALTES, B.P. y BALTES, M. M. (eds.): Succesful aging. Cambridge University Press. New York. pp. 1-34.

FOWLER, J. (1981): Stages of faith. Harper y Row. New York.

GUILDFORD, J. P. (1950): Creativity. American psichologist, 5. 444-454.

- (1959): Traits of creativiy. En H. H. ANDERSON (Ed.) Creativity and is cultivation (pp. 141-161). Harper. N. York.

HEBB, D. O. (1942): The effect of early and late brain injury upon test scores, and nature of normal adult intelligence. Proccedings of the American Pbilosophical Society, 85. pp 275-292.

HENDRICKS, J. (1999): Creativity over the life course. A call for relational perspective. En International Journal of Aging and Human Development, Vol. 48 (2) 85-111.

HERTZOG, C., DIXON, R. A. y HULTSCH, D. F. (1990): Reltionship between metamemory, memory predictions, and memory task performance in adults. Psychology and aging, 5, pp. 215-227. 
HOYER, W. J y RYBASH, J. M. (1994): Characterizing adult cognitive development. Journal of Adult Development, vol.1, $\mathrm{n}^{\circ}$ 1, pp. 7-12.

JOHNSON, S., y THOMAS, R. G. (1992): Technology education and the cognitive revolution. Technology Teacher, 51(4), 1992.

KAUSLER, D. H. (1994): Learning and memory in normal aging. Wiley, New York.

KEGAN, R. G. (1982): The evolving self. Cambrigde Mass. Harvard University Press.

KING, P. (1986): Formal reasoning in adults: a review and critique. En MINES, A. R. y KITCHENER, S. K. (1986): Adult cognitive development. Methods and models Praeger Publishers. New York. pp. 1-21.

KOPLOWITZ, H. (1979): Post-logical thinking. Paper. Harvard International Conference on thinking. Cambridge. M.A. 34.

KRAMER, D.: Post-formal operations? (1983): A need for further conceptualization. Human Development, 26, pp. 91-105.

LABOUVIE-VIEF, G. (1977): Adult cognitive development: IN search of alternative interpretations, Merril-Palmer Quarterly, 24 (4), pp. 227-263.

- (1980): Beyond formal operations: uses and limits of pure logic in life-span development. Human Development, 23, pp. 141-161

- (1984): Logic and self-regulation from youth to maturity: a model. En COMMOMS, M. L.; RICHARDS, F. A.; ARMON, Ch. (Eds.): Beyond formal operations. Late Adolescent and adult cognitive development. Praeger Publishers. New York. pp. 159-179.

- y CHANDLER, R. J. (1978): Cognitive development and life-span developmental theory: idealistic versus contextual perspective. En BALTES, B. P. (ed.):Life-span development and behavior. vol 1. Academic Press. New York.

- y SCHELL, D. A. (1982): Learning and memory in later life. En WOLMAN, B. B. (Ed.). Handbook of developmental psychology. Englewood Cliffs. N.J. Prentice-Hall.

LACHMAN y cols. (1979): Metamemory throughout the adult life span, Developmental Psychology, 15, pp. 543-551.

LIGHT, L. L. (1996): Memory and aging, en E. L. BJORK y R. A. Bjork (Eds.), Memory Handbook of perception and cognition ( $2^{\mathbf{a}}$ de.) (pp. 444-490). Academic Press. San Diego, CA.

MCCRAE, R.; ARENBBERG, D. y COSTA, Jr. P. (1987): Declines in divergent thinking whith age: cross sectional, longitudinal and cross-sequential analysis. Psychology and Aging, $2,130-137$.

MINES, A .R. y KITCHENER, S. K. (1986): Adult cognitive development. Methods and models Praeger Publishers. New York.

MIRAS, M. (1996): Epistemología y psicología genética. Una habitación sin vistas. Anuario de Psicología, pp. 43-51.

PACUAL LEONE, J. (1984): Attention, dialectic, and mental effort: toward and organismic theory of life stages, en COMMOMS, M. L.; RICHARDS, F. A.; ARMON, Ch. (eds.) (1984): Beyond formal operations. Late Adolescent and adult cognitive development. Praeger Publishers. New York. pp. 182-215.

PARK, D. C. y SHAW, R. J. (1992): Effects of environmental suport on implicit and explicit memory in jounger and older adults. Psychology and Aging, 7, 632-642.

PEA, R. W. (1992). Practices of distributed intelligence and designs for education. En G. Salomon (Ed.): Distributed cognition. Cambridge University Press. New York.

PIAGET, J. (1972): Intellectual evolution from adolescence to adulthood Human development, 15, 1-12.

- y INHELDER, B. (1969): The psichology of the child. Basic Books. New York 
ANTONIO VÍCTOR MARTÍN GARCÍA MÁS ALLÁ DE PIAGET: COGNICIÓN ADULTA Y EDUCACIÓN

POON, L. (1985): Differences in human memory with aging: nature, causes, and clinical implications. En J. E. BIRREN y K. W. SCHAIE (Eds.): Handbook of the psychology of aging. Van Nostrand Reinhold. New York.

POON, W. L.; RUBIN, C.D. y WILSON, A. B. (1989): Everday cognition in adulthood and late life. Cambridge University Press.

POZO, J. I. (1996): Maestros y aprendices. Alianza. Madrid.

REBOK, W. G. (1987): Life-span Cognitive Development. CBS College Publishing. New York.

RESNICK, L. B. y otros (Eds.) (1991): Perspectives on socially shared cognition. American Psychological Association. Washington, DC.

RIEGEL, K. F. (1973): Dialectic operations: the final period of cognitive development, Human Development, 16, pp. 346-370.

ROBINSON, A. G. y STERN, S. (1997): Corporate creativity. Berrett-Koehler. San Franciso.

ROGOFF, B. (1990): Apprenticeship in thinking: Cognitive development in social context. Oxford University Press, New York.

ROLFHUS, E. L. y ACKERMAN, P. L. (1996): Self-report knowledge: At the crossroads of ability, interest and personality. Journal of Educational Psychology, 88, pp. 174-188.

RUBIO HERRERA, R. (1990): "El modelo de encapsulación": una alternativa a la interpretación de la cognición adulta. En Revista de Psicología General y Aplicada. 43 (2) pp. 169174.

RUTH, J. E. y BIRREN, J. E. (1985): Creativity in adulthood and old age: Relations to intelligence, sex, and mode of testing. International Journal of Aging and Human Development, 8, 99-110.

RYBASH, J. M; HOYER, W. J. y ROODIN, P. A. (1986): Adult cognition and aging. Pergamon Press. New York.

SALTHOUSE, A. T. (1989): Age-related changes in basic cognitive processes. En VV.AA. (1989): The adult years: Continuity and change. A.P.A. Washington. pp. 9-40.

SASSER-COEN, J. (1993): Qualitative changes in creativity in the second half of life: A lifespan developmental perspective. Journal of Creative Behavior, 27, 18-27.

SCHAIE, K. W. (1979): The primary mental abilities in adulthood: an exploartion of psychometric intelligence. En BALTES, B. P. y BRIM, G. O. (Eds.) (1979): Life span development and behavior, (vol. 2). Academic Press. Nueva York.

SIMONTON, D. K. (1991): Creative productivity throught the adult years. Generations, XV, 1316.

SINNOTT, J. D. (1981): The theory of relativity: A metatheory for development? Human Development, 24, pp. 293-311.

- (1984): Postformal reasoning: the relativistic stage. En COMMONS, M. L; RICHARDS, F. A. y ARMON, C. (Eds.), (1984): Beyond formal operations: Late adolescent and adult cognitive development. Praeger. New York.

- y GUTTMANN, D. (1978): Dialectics of decision makin in older adults. Human Development, 21, (1978), pp. 190-200.

SMITH, A. D. (1980): Age differences in encoding, storage and retrieval. En POON, W.; FOZARD, J. L.; CERMAK, L. S.; STERNBERG. R. J. (1986): Implicit theories of intelligence, creativiy and wisdom. Journal, personality and social psychology, 49, 607-627.

STERNBERG, R. J., Cynthia, A. y otros (Ed.) (1992): Intellectual development. Cambridge University Press. New York.

THURSTONE, L. L. y THURSTONE, T.G . (1946): SRA Primary Mental Abilities. Science Research Associates. Chicago. 
VÁZQUEZ GÓMEZ, G. Y BARCENA, F. (1999): Pedagogía cognitiva: la educación y el estudio de la mente en la Sociedad de la Información. Teoría de la Educación. La Educación y la Cultura en la sociedad de la Información. Webmrte@gugu.usal.es

WECHSLER, D. (1981): Manual for the Wechsler adult intelligence scale revised. The Psychological Corporation. New York.

WELDFORD, A. T. (1969): Age and skill: motor, intellectual, and social, en WELDFORD, A. T. (Ed.) (1969): Interdisciplinary topics in gerontology (4). Decision making and age.: Karger. Basel.

WELLMAN, H. M. y GELMAN, S. A. (1992): Cognitive development: foundations theories of core domains. Annaul Review of psychology, 43, pp. 337-375.

WHITBOURNE, S. (1985): The aging body: Physiological changes and psychological consecuences. Sring-Verlag. New York.

- y WEINSTOCK, C. S. (1986): Adult development. 2nd. Ed. Praeger. New York. 